\title{
PATH FOLLOWING CONTROL OF THE UNDERACTUATED USV BASED ON THE IMPROVED LINE-OF-SIGHT GUIDANCE ALGORITHM
}

\author{
Tao Liu \\ College of Automation, Harbin Engineering University, China \\ Zaopeng Dong \\ Ship Engineering Department, School of Transportation, Wuhan University of Technology, China \\ Hongwang Du \\ College of Automation, Harbin Engineering University, China \\ Lifei Song \\ Ship Engineering Department, School of Transportation, Wuhan University of Technology, China \\ Yunsheng Mao \\ Ship Engineering Department, School of Transportation, Wuhan University of Technology, China
}

\begin{abstract}
The path following control problem of the underactuated unmanned surface vessel (USV) is studied in this paper. An improved line-of-sight (LOS) guidance algorithm is proposed which can adjust adaptively based on the path following error. The global asymptotically stable path following controller is designed based on the nonlinear backstepping method and the Lyapunov stability theory. Firstly, the USV path following error model is established in the Serret-Frenet (SF) coordinate frame. The path following error in the inertial coordinate frame is transformed into the SF coordinate frame, which is used to define the path following control problem. Secondly, inspired by the traditional LOS guidance algorithm, the longitudinal path following error in the SF coordinate frame is introduced into the improved LOS guidance algorithm. This allows the algorithm to adjust adaptively to the desired path. Thirdly, in order to solve the underactuated problem of the USV path following control system, the tangential velocity of the desired path is designed as a virtual input. The underactuated problem is converted to a virtual fully actuated problem by designing the virtual control law for the tangential velocity. Finally, by combining backstepping design principles and the Lyapunov stability theory, the longitudinal thrust control law and the yaw torque control law are designed for the underactuated USV. Meanwhile, the global asymptotic stability of the path following error is proved. Simulation experiments demonstrate the effectiveness and reliability of the improved LOS guidance algorithm and the path following controller.
\end{abstract}

Keywords: unmanned surface vessel (USV); path following control; Serret-Frenet coordinate frame; underactuated system; improved lineof-sight guidance algorithm; nonlinear backstepping method

\section{INTRODUCTION}

Unmanned surface vessels (USV) are attracting more and more attention from researchers all over the world because of their extensive applications in military reconnaissance, homeland security, shallow water surveys, environmental monitoring, and in coordinating the operation with autonomous underwater vehicles (AUV) [1-3]. The USV path following control problem is an active field of studies, due to its theoretical challenges and important applications. The main difficulty with controlling the underactuated USV is that only the yaw and surge axes are directly actuated, while the sway axis remains non-actuated. This configuration is by far most common among USVs. Moreover, the underactuated USVs are a class of underactuated mechanical systems with non-integrable dynamics which cannot be transformed into a driftless system. The application of the Brockett's theorem proves the absence of pure state feedbacks that would be able to asymptotically stabilize the underactuated USV at a fixed point. Thus, the underactuated USV cannot be stabilized by the time-invariant continuous control law, although it is an open-loop controllable device. Several authors have contributed a set of novel ideas and strategies, mainly including discontinuous feedback and time-varying feedback methods. Due to high dependence on the reference model and complicated trajectory tracking controllers, the path following problem, referred to as forcing an underactuated USV to follow a specified path at a desired forward speed without a time constraint, is widely used in practice. Below is a brief review of existing works on path following control 
of underactuated USVs and ships that has motivated the authors to write this paper.

A nonlinear robust adaptive control strategy which forces an underactuated surface ship to follow the predefined path at the desired speed is developed in [4], but several assumptions made in the paper allow the controller only to have the ship follow some certain paths. Path following control of an underactuated autonomous vehicle is addressed in [5], where the authors demonstrate the way in which adaptive switching supervisory control can be combined with the nonlinear Lyapunov based tracking control law to solve the problem of global boundedness and make the position tracking error to converge to a vicinity of the origin that can be made arbitrarily small. The point to point navigation of an underactuated ship is considered in [6], and the model predictive control (MPC) for waypoint tracking of an underactuated surface vessel with input constraints is presented in [7]. Path following control of snake robots along straight paths is considered in [8]. Under the assumption that the forward velocity of the snake robot is nonzero and positive, the authors prove that the proposed path following controller k-exponentially stabilizes the snake robot to any desired straight path. The problem of robust adaptive path following control for an underactuated ship with model uncertainties and nonzero-mean time-varying disturbances is studied in [9], where a concise adaptive neural network (NN) based control scheme is proposed. This scheme makes use of backstepping, feedforward approximations, dynamic surface control, and minimal learning parameter techniques. Starting from the waypoint based path following control for marine ships, a novel dynamic virtual ship (DVS) guidance principle is developed in [10] after implementing the assumption that "the reference path is generated using a virtual ship", which is critical for applying these theoretical studies in practice. The problem of robust adaptive path following control for uncertain underactuated ships in the waypoint based navigation, which is a field of marine practice, is studied in [11]. A novel logical virtual ship (LVS) based steering law is developed to program the rational reference route which is then used to guide an underactuated ship in practical engineering. A level curve approach is introduced to design global path following controllers for an underactuated surface ship in [12], where the controllers are designed based on the Lyapunov direct and backstepping methods to make the ship position to satisfy the path equation and to move the ship along the path tangentially. An optimal controller is proposed for the integrated path following and roll motion control problem in [13], where the line-ofsight (LOS) technique is used to extend straight-line path following to arbitrary path following. A nonlinear adaptive path following controller which can compensate drift forces through vehicle sideslip is presented in [14]. In that paper the proposed algorithm, motivated by the LOS guidance principle used by ancient navigators, is extended to Dubins path following. A saturated tracking controller for underactuated autonomous marine surface vehicles with limited torque is proposed in [15], where a dynamic second-order open-loop error model is developed in the actuated degrees of freedom to simplify the design procedure. A state feedback based backstepping control algorithm is used in [16] for solving the trajectory tracking problem of an underactuated USV in the horizontal plane. In that paper the well known persistent exciting (PE) condition of yaw velocity is completely relaxed. A design of new controllers that make underactuated ships navigating under both deterministic and stochastic sea loads to globally track the reference path is presented in [17], where the loads are decomposed to a deterministic part, treated as a set of unknown constants, and a time-varying part, which is considered a form of stochastic disturbances with unknown time-varying covariances. The path following problem of an underactuated AUV is addressed in [18], with the aim of dealing with parameter uncertainties and current disturbances. In that paper, an adaptive robust control system is developed by employing fuzzy logic, backstepping, and the sliding mode control theory. Tracking geometric paths in output spaces of nonlinear systems subject to input and state constraints without pre-specified timing requirements, commonly referred to as the constrained output path following problem, is considered in [19], where a predictive control approach is proposed to constrained path following problems with and without velocity assignments. The path following control problem for an unmanned marine surface vessel in the presence of uncertainties and input saturation is presented in [20], where the backstepping technique, augmented by a robust adaptive radial basis function neural network (RBFNN) and an auxiliary design system, is employed as the main control framework. A two-dimensional path following control system for autonomous surface vehicles is presented in [21], where the guidance system is obtained through a waypoint guidance scheme based on a vector field algorithm.

In the above papers addressing the underactuated ship/ USV path following control problem, some results have been obtained under assumptions which allow the controller only to have the ship follow a certain path $[5,8]$. Moreover, some of the published research results have been derived by combining the traditional LOS approach and nonlinear control methods $[7,13,14,20$,$] . It is noteworthy that some researchers execute$ path following control using complex system state transform techniques and intelligent learning algorithms which are difficult to design and not easy to implement in the real path following control system [4,6, 9-12,15-19,21]. Motivated by those considerations, this paper addresses path following control of an underactuated USV based on an improved LOS guidance algorithm. Compared with the existing literature, the main contributions of the paper are as follows: (i) a novel modified LOS guidance algorithm is proposed for path following control of the underactuated USV, which can adaptively change the guidance law to respond to longitudinal and/or lateral path following error changes; (ii) the USV velocity component which is tangent to the desired path is selected as the virtual input, after which the underactuated system is transformed into a virtual fully actuated system which makes the path following controller easier to design; (iii) the longitudinal path following error is introduced into the path following controller, which increases controller's convergence speed and stability; (iv) designing the path following controller is simpler than the abovementioned 
controllers, moreover the controller is easier to implement and its global uniform asymptotic stability is good; (v) the well known persistent exciting (PE) condition is completely released in the path following controller proposed in this paper; (vi) the designed controller can execute USV path following control without the use of side thrusters, thus obtaining the underactuated USV control, and the global stability of the overall path following control system is proved by the Lyapunov theory and the Lasalle invariance principle.

The reminder of the paper is organized as follows. Firstly, the underactuated USV model is established and the USV path following control problem is formulated. Secondly, a novel improved LOS guidance algorithm is proposed and the path following controller is designed. Thirdly, global uniform asymptotic stability of the USV path following controller is proved based on the Lyapunov theory and the Lasalle invariance principle. The results of simulation experiments of the underactuated USV in a semi physical simulation platform are presented and, finally, conclusions are given.

\section{PROBLEM FORMULATION}

In this section, the problem of path following control of an underactuated USV in the horizontal plane is formulated. The kinematic and dynamic equations of the USV are presented in step 1, and the path following control problem for the underactuated USV is formulated in step 2 .

\section{Step 1: USV modeling}

A three-degree-of-freedom underactuated USV in the horizontal plane is considered. The state of the USV is given by the vector $\left[\boldsymbol{\eta}^{\mathrm{T}}, \boldsymbol{v}^{\mathrm{T}}\right]^{\mathrm{T}}$ where $\boldsymbol{\eta}=[x, y, \varphi]^{\mathrm{T}}$ describes the USV position and the orientation of the USV with respect to the inertial frame $\{I\}-x o y$ as show in Fig. 1. In particular, $x$ and $y$ are Cartesian coordinates of the USV's center of mass $o_{b}$ in $\{I\}, \varphi$ denotes the USV yaw angle or heading of the USV. The vector $\tilde{\mathbf{o}}=[u, v, r]^{\mathrm{T}}$ contains linear and angular velocities of the USV defined in the body-fixed frame $\{b\}$, where $u$ is the surge velocity, $v$ is the sway velocity and $r$ is the yaw rate.

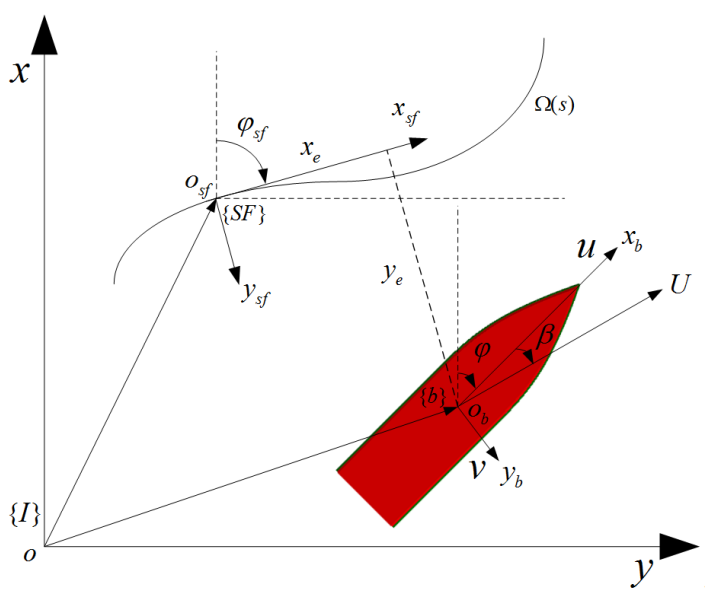

Fig.1. Coordinate frame of USV path following system
According to the literature [22], the kinematic and dynamic equations of the underactuated USV in the horizontal plane can be expressed as:

$$
\dot{\boldsymbol{\eta}}=\mathbf{R}(\varphi) \mathbf{v}
$$

with

$$
\mathbf{R}(\varphi)=\left[\begin{array}{ccc}
\cos (\varphi) & -\sin (\varphi) & 0 \\
\sin (\varphi) & \cos (\varphi) & 0 \\
0 & 0 & 1
\end{array}\right]
$$

and

$$
\mathbf{M i}+\mathbf{C}(\boldsymbol{v}) \boldsymbol{v}+\mathbf{D v}=\boldsymbol{\tau}
$$

with

$\mathbf{M}=\operatorname{diag}\left\{m_{11}, m_{22}, m_{33}\right\}, \mathbf{D}=\operatorname{diag}\left\{d_{11}, d_{22}, d_{33}\right\}, \mathbf{C}(\boldsymbol{v})=\left[\begin{array}{ccc}0 & 0 & -m_{22} v \\ 0 & 0 & m_{11} u \\ m_{22} v & -m_{11} u & 0\end{array}\right], \boldsymbol{\tau}=\left[\begin{array}{c}\tau_{u} \\ 0 \\ \tau_{r}\end{array}\right]$.

where $\tau_{u}$ and $\tau_{r}$ are the surge force and the yaw moment, $m_{11} 、 m_{22} 、 m_{33}$ are the USV's inertia coefficients including added mass effects and $d_{11} 、 d_{22} 、 d_{33}$ are the hydrodynamic damping coefficients.

\section{Step 2: Problem Formulation}

As shown in Fig. 1, $\Omega(s)$ is the desired path which needs to be followed in the inertial frame $\{I\}-x o y$, and $S$ is the parameter of $\Omega(s)$. Point $o_{s f}$ is the target reference point on the path, established in the Serret-Frenet (SF) coordinate frame $\{S F\}-x_{s f} o_{s f} y_{s f}$, the origin of which is chosen to coincide with ${ }_{s f}$, as shown in Fig. 1. The $x_{s f}$-axis direction is tangential direction and the $y_{s f}$-axis direction is normal to $\Omega(s)$ at point $o_{s f}, \varphi_{s f}$ is the angle between the $x$-axis and the $x_{s f}$-axis. As shown in Fig. $1, U=\sqrt{u^{2}+v^{2}}$, and $\beta$ is the drift angle between $U$ and $x_{b}$-axis direction. Coordinate values of the USV's center of mass $o_{b}$ in SF coordinate frame $\{S F\}$ $x_{s f} o_{s f} y_{s f}$ are $\left(x_{e}, y_{e}\right)$, and they are path following errors. The following equations can be easily obtained, see Fig. 1:

$$
\left[\begin{array}{c}
x_{e} \\
y_{e} \\
\varphi_{e}
\end{array}\right]=\left[\begin{array}{ccc}
\cos \left(\varphi_{s f}\right) & \sin \left(\varphi_{s f}\right) & 0 \\
-\sin \left(\varphi_{s f}\right) & \cos \left(\varphi_{s f}\right) & 0 \\
0 & 0 & 1
\end{array}\right]\left[\begin{array}{c}
x-x_{s f} \\
y-y_{s f} \\
\varphi+\beta-\varphi_{s f}
\end{array}\right]
$$

Differentiating both sides of (3) leads to the following path following error system:

$$
\left\{\begin{array}{l}
\dot{x}_{e}=K(s) \dot{s} y_{e}-\dot{s}+U \cos \left(\varphi_{e}\right) \\
\dot{y}_{e}=-K(s) \dot{s} x_{e}+U \sin \left(\varphi_{e}\right) \\
\dot{\varphi}_{e}=r+\dot{\beta}-K(s) \dot{s}
\end{array}\right.
$$

where $\beta=\arctan (v / u), \dot{s}$ is the virtual tangential velocity of the desired path, and $K(s)$ is the path curvature at the reference target point. 
The general path following control problem of the underactuated USV considered in this paper can be formulated as follows:

Let us design the control laws of $\tau_{u}$ and $\tau_{r}$ for the path following control system, which will make the path following errors $p_{e}=\left[x_{e}, y_{e}, \varphi\right]^{\mathrm{T}}$ converge to zero and the velocity $u$ converge to desired velocity $u_{d}$.

\section{CONTROLLER DESIGN}

Step 1: designing control laws for $\tau_{u}$ and $\tau_{r}$

Based on the traditional LOS method, the improved design of the look ahead distance has the following form:

$$
\Delta=\frac{\sqrt{\lambda_{1}+\left(\lambda_{2} x_{e}\right)^{2}}}{\lambda_{3}}
$$

and the improved LOS guidance law can be written as:

$$
\varphi_{\text {los }}=-\arctan \left(\frac{y_{e}}{\Delta}\right)=-\arctan \left[\frac{\lambda_{3} y_{e}}{\sqrt{\lambda_{1}+\left(\lambda_{2} x_{e}\right)^{2}}}\right]
$$

where $\lambda_{1} 、 \lambda_{2} 、 \lambda_{3}$ are positive parameters. The improved LOS guidance law can adaptively change when the reference target point changes.

Let us introduce the following new variables:

$$
\left\{\begin{aligned}
\Theta_{1} & =u-u_{d} \\
\Theta_{2} & =\varphi_{e}-\varphi_{l o s}=\varphi-\varphi_{s f}+\beta-\varphi_{l o s} \\
\Theta_{3} & =\dot{\Theta}_{2}=\dot{\varphi}_{e}-\dot{\varphi}_{l o s}=\dot{\varphi}-\dot{\varphi}_{s f}+\dot{\beta}-\dot{\varphi}_{l o s} \\
& =r-\dot{\varphi}_{s f}+\dot{\beta}-\dot{\varphi}_{l o s}
\end{aligned}\right.
$$

where $u_{d}$ is the desired velocity. Differentiating both sides of expression (7) leads to:

$$
\left\{\begin{array}{l}
\dot{\Theta}_{1}=\frac{m_{22}}{m_{11}} v r-\frac{d_{11}}{m_{11}} u+\frac{1}{m_{11}} \tau_{u}-\dot{u}_{d} \\
\dot{\Theta}_{2}=\Theta_{3} \\
\dot{\Theta}_{3}=\frac{m_{11}-m_{22}}{m_{33}} u v-\frac{d_{33}}{m_{33}} r+\frac{1}{m_{33}} \tau_{r}-\dot{\varphi}_{s f}+\dot{\beta}-\dot{\varphi}_{l o s}
\end{array}\right.
$$

Based on the first expression of (8), the control law for $\tau_{u}$ can be designed in the form:

$$
\tau_{u}=-m_{22} v r+d_{11} u+m_{11} \dot{u}_{d}-k_{1} m_{11} \Theta_{1}
$$

where $k_{1}$ is a positive parameter. Based on the second and third expressions of (8), the control law for $\tau_{r}$ can be designed as:

$\tau_{r}=-\left(m_{11}-m_{22}\right) u v+d_{33} r-k_{2} m_{33} \Theta_{2}-k_{3} m_{33} \Theta_{3}+m_{33}\left(\dot{\varphi}_{s f}-\dot{\beta}+\dot{\varphi}_{l o s}\right)$

where $k_{2} 、 k_{3}$ are both positive parameters.

\section{Step 2: designing control law for $\dot{S}$}

The variable $\Theta_{2}$ convergences to zero under the control laws (9) and (10), as will be proved in the next section, step 1. The first expression of (4) can be rewritten as follows, see reference on the Lasalle invariance principle in [23]:

$$
\begin{aligned}
\dot{x}_{e} & =K(s) \dot{s} y_{e}-\dot{s}+U \cos \left(\varphi_{\text {los }}\right) \\
& =K(s) \dot{s} y_{e}-\dot{s}+U \frac{\sqrt{\lambda_{1}+\left(\lambda_{2} x_{e}\right)^{2}}}{\sqrt{\lambda_{1}+\left(\lambda_{2} x_{e}\right)^{2}+\left(\lambda_{3} y_{e}\right)^{2}}}
\end{aligned}
$$

By analyzing the above expression (11), we can design the virtual control law for $\dot{s}$ as follows:

$$
\begin{aligned}
\dot{s} & =U \frac{\sqrt{\lambda_{1}+\left(\lambda_{2} x_{e}\right)^{2}}}{\sqrt{\lambda_{1}+\left(\lambda_{2} x_{e}\right)^{2}+\left(\lambda_{3} y_{e}\right)^{2}}}+\lambda_{4} x_{e} U \frac{1}{\sqrt{\lambda_{1}+\left(\lambda_{2} x_{e}\right)^{2}+\left(\lambda_{3} y_{e}\right)^{2}}} \\
& =\frac{\left[\sqrt{\lambda_{1}+\left(\lambda_{2} x_{e}\right)^{2}}+\lambda_{4} x_{e}\right] U}{\sqrt{\lambda_{1}+\left(\lambda_{2} x_{e}\right)^{2}+\left(\lambda_{3} y_{e}\right)^{2}}}
\end{aligned}
$$

where $\lambda_{4}$ is positive parameter.

\section{STABILITY ANALYSIS}

Step 1: stability analysis of $\Theta_{1} 、 \Theta_{2} 、 \Theta_{3}$.

Substituting expressions (9) and (10) into expression (8) yields:

$$
\left\{\begin{array}{l}
\dot{\Theta}_{1}=-k_{1} \Theta_{1} \\
\dot{\Theta}_{2}=\Theta_{3} \\
\dot{\Theta}_{3}=-k_{2} \Theta_{2}-k_{3} \Theta_{3}
\end{array}\right.
$$

We consider the following Lyapunov function candidate $V_{1}$ of the system (13):

$$
V_{1}=\frac{1}{2} \Theta_{1}^{2}+\frac{1}{2} k_{2} \Theta_{2}^{2}+\frac{1}{2} \Theta_{3}^{2}
$$

Differentiating both sides of (14) along the solutions of the system (13) leads to:

$$
\begin{aligned}
\dot{V}_{1} & =\Theta_{1} \dot{\Theta}_{1}+k_{2} \Theta_{2} \dot{\Theta}_{2}+\Theta_{3} \dot{\Theta}_{3} \\
& =-k_{1} \Theta_{1}^{2}+k_{2} \Theta_{2} \Theta_{3}-k_{2} \Theta_{2} \Theta_{3}-k_{3} \Theta_{3}^{2} \\
& =-k_{1} \Theta_{1}^{2}-k_{3} \Theta_{3}^{2} \\
& \leq 0
\end{aligned}
$$

It can be proved, by combining expression (14) and (15) and applying the Lyapunov stability theory, that $\Theta_{1} 、 \Theta_{2} 、 \Theta_{3}$ converge to zero as $t \rightarrow \infty$. 
Step 2: stability analysis of $x_{e} 、 y_{e}$.

Substituting expression (12) into (11) leads to:

$$
\dot{x}_{e}=K(s) \dot{s} y_{e}-\frac{\lambda_{4} x_{e} U}{\sqrt{\lambda_{1}+\left(\lambda_{2} x_{e}\right)^{2}+\left(\lambda_{3} y_{e}\right)^{2}}}
$$

As was proved in step $1 \Theta_{2}$ convergence to zero, see reference on the Lasalle invariance principle in [23]. Consequently the second expression of (4) can be rewritten as follows:

$$
\begin{aligned}
\dot{y}_{e} & =-K(s) \dot{s} x_{e}+U \sin \left(\varphi_{\text {los }}\right) \\
& =-K(s) \dot{s} x_{e}-\frac{\lambda_{3} y_{e} U}{\sqrt{\lambda_{1}+\left(\lambda_{2} x_{e}\right)^{2}+\left(\lambda_{3} y_{e}\right)^{2}}}
\end{aligned}
$$

Combining the above expressions (16) and (17) leads to the following underactuated USV path following error system:

$$
\left\{\begin{array}{l}
\dot{x}_{e}=K(s) \dot{s} y_{e}-\frac{\lambda_{4} x_{e} U}{\sqrt{\lambda_{1}+\left(\lambda_{2} x_{e}\right)^{2}+\left(\lambda_{3} y_{e}\right)^{2}}} \\
\dot{y}_{e}=-K(s) \dot{s} x_{e}-\frac{\lambda_{3} y_{e} U}{\sqrt{\lambda_{1}+\left(\lambda_{2} x_{e}\right)^{2}+\left(\lambda_{3} y_{e}\right)^{2}}}
\end{array}\right.
$$

We consider the following Lyapunov function candidate $V_{2}$ of the system (18):

$$
V_{2}=\frac{1}{2} x_{e}^{2}+\frac{1}{2} y_{e}^{2}
$$

Differentiating both sides of (19) along the solutions of the system (18) results in:

$$
\begin{aligned}
\dot{V}_{2} & =x_{e} \dot{x}_{e}+y_{e} \dot{y}_{e} \\
& =K(s) \dot{s} x_{e} y_{e}-\frac{\lambda_{4} x_{e}^{2} U}{\sqrt{\lambda_{1}+\left(\lambda_{2} x_{e}\right)^{2}+\left(\lambda_{3} y_{e}\right)^{2}}}-K(s) \dot{s} x_{e} y_{e}-\frac{\lambda_{3} y_{e}^{2} U}{\sqrt{\lambda_{1}+\left(\lambda_{2} x_{e}\right)^{2}+\left(\lambda_{3} y_{e}\right)^{2}}} \\
& =-\frac{\lambda_{4} U x_{e}^{2}+\lambda_{3} U y_{e}^{2}}{\sqrt{\lambda_{1}+\left(\lambda_{2} x_{e}\right)^{2}+\left(\lambda_{3} y_{e}\right)^{2}}} \\
& \leq 0
\end{aligned}
$$

It can be proved, by combining expressions (19) and (20) and applying the Lyapunov stability theory, that $x_{e}, y_{e}$, and $\varphi_{\text {los }}$ converge to zero as $t \rightarrow \infty$. As $\Theta_{1}$ converges to zero, the velocity $u$ converges to the desired velocity $u_{d}$. As $\Theta_{2}$ and $\varphi_{\text {los }}$ both converge to zero, then $\varphi_{e}$ also converges to zero.

Above all, using the control laws for $\tau_{u}$ and $\tau_{r}$ in (9) and (10) in combination with the virtual control law for $\dot{s}$ in (12), the underactuated USV path following control system makes the path following errors $p_{e}=\left[x_{e}, y_{e}, \varphi\right]^{\mathrm{T}}$ converge to zero and the velocity $u$ converge to the desired velocity $u_{d}$..

\section{SIMULATION EXPERIMENTS}

In order to verify and illustrate the effectiveness of the path following control algorithm proposed for the underactuated USV, a series of computer simulation experiments have been carried out in the semi physical simulation platform on a USV model with the following hydrodynamic parameters: $m_{11}=25.8, m_{22}=33.8, m_{33}=2.8, \quad d_{11}=27, d_{22}=17$, $d_{33}=0.5$. The parameters of the designed path following controller were chosen as: $k_{1}=10, k_{2}=20, k_{3}=20, \lambda_{1}=10$ $\lambda_{2}=0.5, \lambda_{3}=1, \lambda_{4}=20$. Two cases were analyzed:

Case 1: The desired path and the initial state of the USV are chosen as: $x_{s f}=50 \cos (0.02 s), y_{s f}=50 \sin (0.02 s)$, $x(0)=y(0)=\varphi(0)=0, \quad u(0)=0.5 \mathrm{~m} / \mathrm{s}, \quad v(0)=r(0)=0$. The desired velocity $u_{d}$ is chosen as: $u_{d}=5 \mathrm{~m} / \mathrm{s}$. The obtained simulation results are shown in Figs. 2-3.

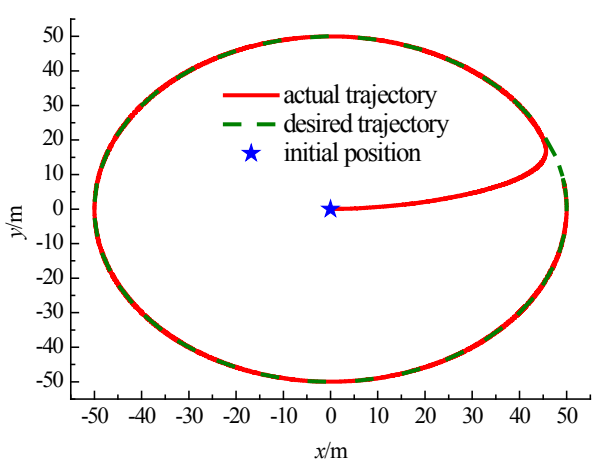

Fig.2. USV curved path following control trajectory

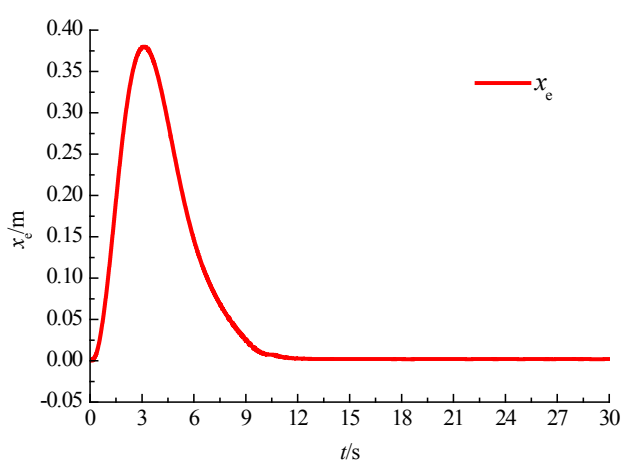

(a) Longitudinal position error convergence curve

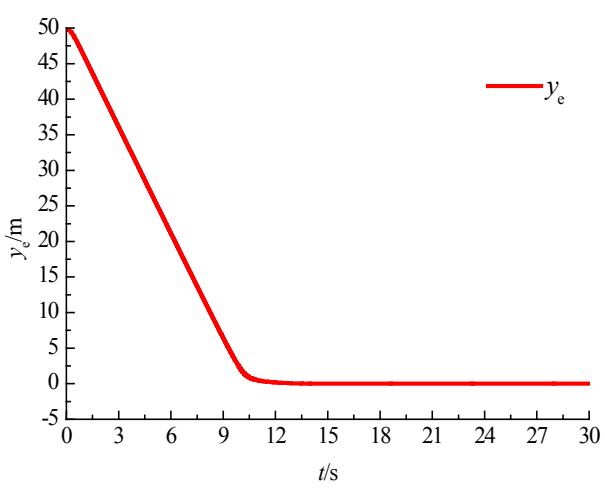

(b) Lateral position error convergence curve 


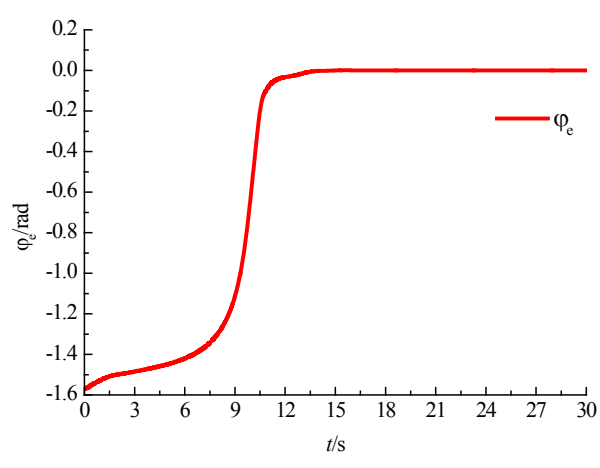

(c) Heading angle error convergence curve

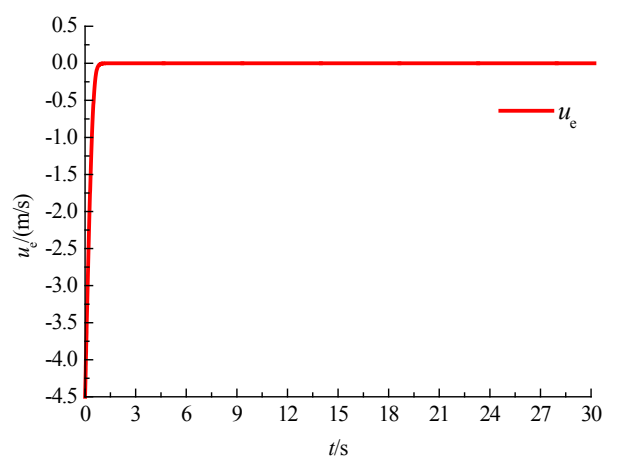

(d) Longitudinal velocity convergence curve

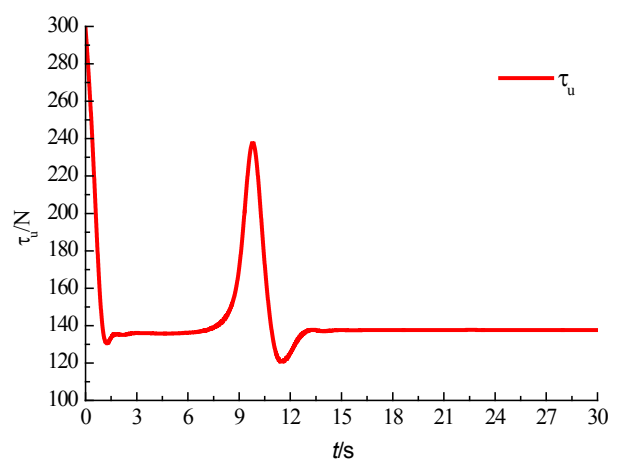

(e) Longitudinal thrust control input curve

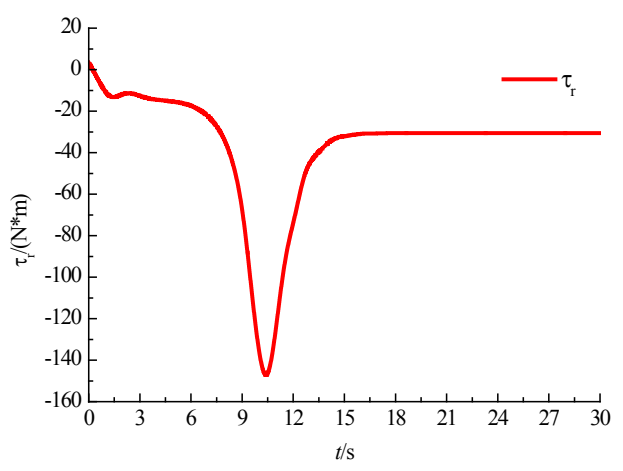

(f) Yaw moment control input curve

Fig.3. Experiment results of USV's curve path following control
Case 2: The desired path is chosen as: $x_{s f}=\sqrt{2} s / 2$ , $y_{s f}=\sqrt{2} s / 2$, and the initial state of the USV is chosen as: $x(0)=20 \mathrm{~m}, y(0)=40 \mathrm{~m}, \varphi(0)=0, u(0)=0.5 \mathrm{~m} / \mathrm{s}$, $v(0)=0, r(0)=0$. The desired velocity $u_{d}$ is chosen as: $u_{d}=5 \mathrm{~m} / \mathrm{s}$. The obtained simulation results are shown in Figs 4-5.

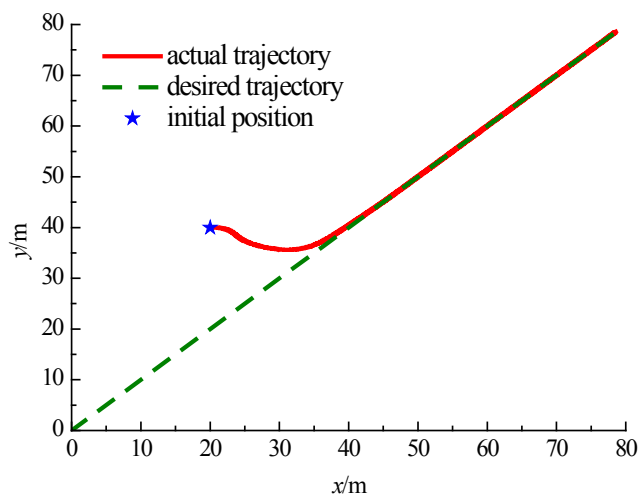

Fig.4 USV's straight-line path following control trajectory

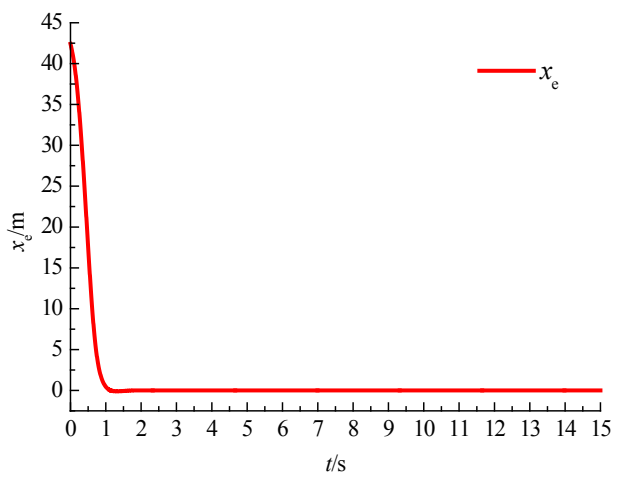

(a) Longitudinal position error convergence curve

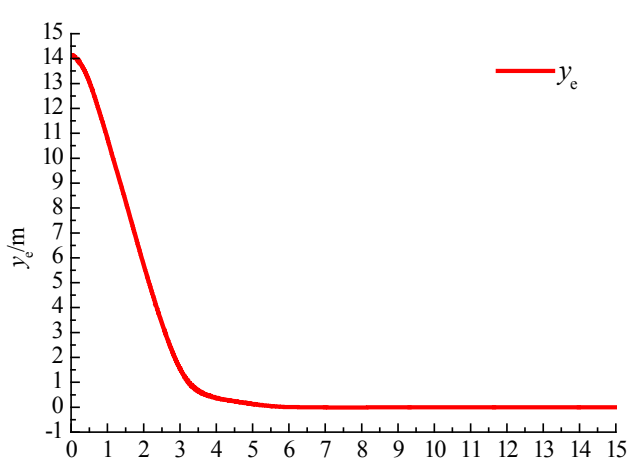

$t / \mathrm{s}$

(b) Lateral position error convergence curve 


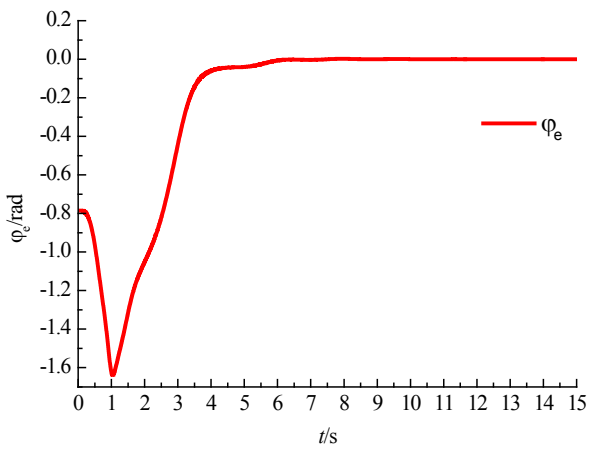

(c) Heading angle error convergence curve

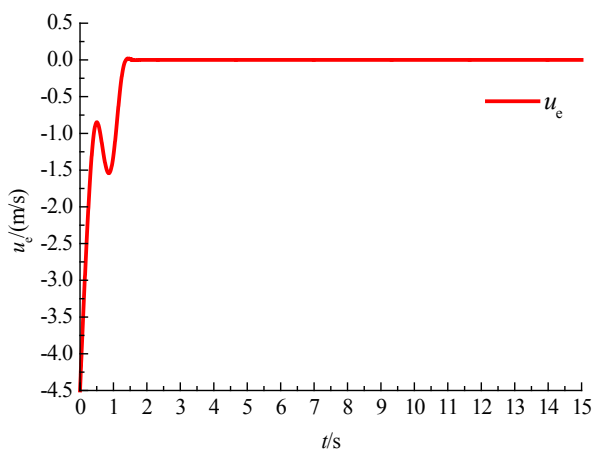

(d) Longitudinal velocity convergence curve

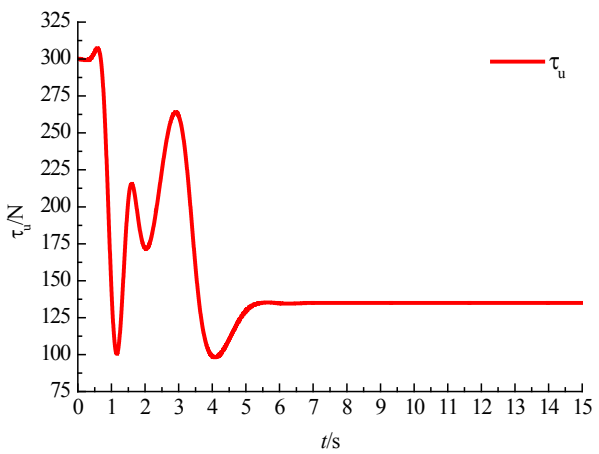

(e) Longitudinal thrust control input curve

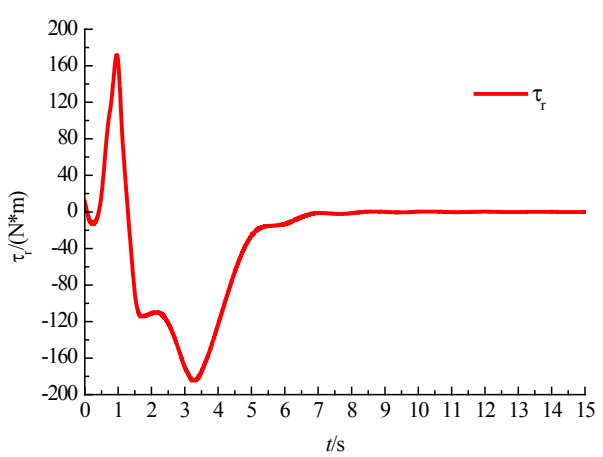

(f) Yaw moment control input curve

Fig.5 Simulated results of USV straight-line path following control
It can be observed in the results of the two cases of simulation experiments that the path following control of the underactuated USV is executed quickly, effectively and reliably by the path following controller, designed based on the above proposed improved LOS guidance algorithm. The fluctuations appearing during the longitudinal control force and yaw moment convergence process, see relevant curves in Figs. 3 and 5, most likely result from large course changes introduced by the controller before the USV is driven into the desired path. These course changes affect the angular and lateral velocities. The longitudinal thrust and the yaw moment are sensitive to angular and lateral velocity changes, and this leads to certain control force and/or moment fluctuations as a response to the abovementioned course and speed changes. The control force and moment also reach a stable state when the course and speed become stable.

Analyzing the obtained experimental results on the background of the available literature reveals the following advantages of the designed controller: Compared with the literature $[7,8,11,14]$, the path convergence speed of the path following control method is faster, the convergence performance before entering the path is better, and the overshoot is smaller. Compared with the literature $[9,10,12,16]$, the vibration of the controller is relatively smaller and the stability is better. Compared with the path following controller designed in the literature $[5,19,21]$, the present controller is more versatile and can realize both the curve path and the straight-line path following control. Summarizing the above analysis, we can get the following results:

1. Compared with other existing results, the designed path following controller can execute path following control of an underactuated USV faster and in a more effective, stable, and reliable way.

2. The speed of convergence and the stability of the system are improved by the USV path following controller designed based on the improved LOS guidance algorithm, as a result of introducing the longitudinal path following error into it.

3. The designed path following controller has global symptotic stability and can execute path following control of the underactuated USV quickly and effectively, at the same time maintaining the stability of the system during the entire process.

4. The path following control method presented in the paper is more versatile, and can execute both the curve path and the straight-line path following control.

\section{CONCLUSIONS}

Based on the traditional LOS guidance algorithm, the longitudinal path following error in the SF coordinate system is presented and the improved LOS guidance algorithm which can automatically adapt to the desired path is proposed, with an intention to improve the speed of convergence of the state variables and the control performance of the controller. The tangent velocity of the reference target in the desired path has been selected as the virtual control input, and the 
underactuated USV path following control problem was converted to a virtual fully actuated control problem. As a result, the process of designing the path following controller is simplified and the complex transformation design of the system model is avoided. The underactuated USV path following controller, which has global asymptotic stability, is designed based on nonlinear backstepping and the Lyapunov stability theory, and can execute path following control in a quick and stable way. Compared with path following controllers presented in the literature, the present controller has faster convergence speed and better control performance. In addition, the path following control method designed in the paper is more versatile, as it can execute both the curve path and the straight-line path following control. Considering that the USV is always disturbed by wind, waves, and ocean currents, path following control of the USV in the presence of the external environment interference will be the focus of future studies by the authors.

\section{ACKNOWLEDGMENTS}

This work was supported by the National Natural Science Foundation of China (Grant Nos. 51409054, 51409059, 51409061, 51509057 and 51579022) and the National High Technology Research and Development Program 863 of PR China (No. 2014AA09A509).

\section{BIBLIOGRAPHY}

1. Sohn S.I., Oh J.H., Lee Y.S., et al. Design of a full-cellpowered catamaran-type unmanned surface vehicle. IEEE Journal of Oceanic Engineering, 2015, 40(2): 388-396.

2. Dong Z.P., Wan L., Liu T., et al. Horizontal-plane trajectory-tracking control of an unmanned maritime vehicle in the presence of ocean currents. International Journal of Advanced Robotic Systems, 2016, 13: 83, 1-14.

3. Larrazabal J.M., Penas M.S. Intelligent rudder control of an unmanned surface vessel. Expert Systems with Applications, 2016, 55: 106-117.

4. Do K.D., Jiang Z.P., Pan J. Robust adaptive path following of underactuated ships. Automatica, 2004, 40(6): 929-944.

5. Aguiar A.P., Hespanha J.P. Trajectory-tracking and pathfollowing of underactuated autonomous vehicles with parametric modeling uncertainty. IEEE Transactions on Automatic Control, 2007, 52(8): 1362-1379.

6. Li J.H., Lee P.M., Jun B.H., Lim Y.K. Point-to-point navigation of underactuated ships. Automatica, 2008, 44(12): 3201-3205.
7. Oh S.R., Sun J. Path following of underactuated marine surface vessels using line-of-sight based model predictive control. Ocean Engineering, 2010, 37(2-3): 289-295.

8. Liljeback P., Haugstuen I.U., Pettersen K.Y. Path following control of planar snake robots using a cascaded approach. IEEE Transactions on Control Systems Technology, 2012, 20(1): 111-126.

9. Zhang G.Q., Zhang X.K. Concise robust adaptive pathfollowing control of underactuated ships using DSC and MLP. IEEE Journal of Oceanic Engineering, 2014, 39(4): 685-694.

10. Zhang G.Q., Zhang X.K. A novel DVS guidance principle and robust adaptive path-following control for underactuated ships using low frequency gain-learning. ISA Transactions, 2015, 56: 75-85.

11. Zhang G.Q., Zhang X.K., Zheng Y.F. Adaptive neural path-following control for underactuated ships in fields of marine practice. Ocean Engineering, 2015, 104: 558-567.

12. Do K.D. Global path-following control of stochastic underactuated ships: a level curve approach. Journal of Dynamic Systems Measurement and Control-Transactions of the ASME, 2015, 137(7): 071010, 1-10.

13. Liu C., Sun J., Zou Z.J. Integrated line of sight and model predictive control for path following and roll motion control using rudder. Journal of Ship Research, 2015, 59(2): 99-112.

14. Fossen T.I., Pettersen K.Y., Galeazzi R. Line-of-sight path following for dubins paths with adaptive sideslip compensation of drift forces. IEEE Transactions on Control Systems Technology, 2015, 23(2): 820-827.

15. Shojaei K. Neural adaptive robust control of underactuated marine surface vehicles with input saturation. Applied Ocean Research, 2015, 53: 267-278.

16. Dong Z.P., Wan L., Li Y.M., et al. Trajectory tracking control of underactuated USV based on modified backstepping approach. International Journal of Naval Architecture and Ocean Engineering, 2015, 7(5): 817-832.

17. Do K.D. Global robust adaptive path-tracking control of underactuated ships under stochastic disturbances. Ocean Engineering, 2016, 111: 267-278.

18. Liang X., Wan L., Blake J.I.R., et al. Path following of an underactuated AUV based on fuzzy backstepping sliding mode control. International Journal of Advanced Robotic Systems, 2016, 13: 122, 1-11. 
19. Faulwasser T., Findeisen R. Nonlinear model predictive control for constrained output path following. IEEE Transactions on Automatic Control, 2016, 61(4): 1026-1039.

20. Zheng Z.W., Sun L. Path following control for marine surface vessel with uncertainties and input saturation. Neurocomputing 2016, 177: 158-167.

21. Xu H.T., Soares C.G. Vector field path following for surface marine vessel and parameter identification based on LS-SVM. Ocean Engineering, 2016, 113: 151-161.

22. Fossen T.I. Handbook of marine craft hydrodynamics and motion control. New York: John Wiley \& Sons. 2011.

23. Isidori, A. Nonlinear control systems. Berlin: Springer Science \& Business Media, 1995.

\section{CONTACT WITH THE AUTHOR}

\author{
Zaopeng Dong \\ e-mail:naval_architecture@163.com
}

Ship Engineering Department

School of Transportation, Wuhan University of Technology Heping Road, 430000 Wuhan

China 